\title{
Design of a Femtosecond Ti:sapphire Laser for Generation and Temporal Optimization of 0.5-PW Laser Pulses at a 0.1-Hz Repetition Rate
}

\author{
Jae Hee Sung*, Tae Jun Yu, Seong Ku Lee, Tae Moon Jeong, II Woo Choi, \\ Do-Kyeong Ko, and Jongmin Lee ${ }^{\dagger}$ \\ Center for Femto-Atto Science and Technology $\&$ Advanced Photonics Research Institute (APRI), \\ GIST, Gwangju 500-712, Korea
}

(Received January 5, 2009 : revised February 4, 2009 : accepted February 6, 2009)

\begin{abstract}
A chirped-pulse amplification Ti:sapphire laser system has been designed using a 10-Hz 100-TW Ti:sapphire laser to generate 0.1-Hz 0.5-PW laser pulses and optimize their temporal qualities such as temporal contrast and pulse duration. A high-energy booster amplifier to be added is expected to produce an energy above $30 \mathrm{~J}$ through the parasitic lasing suppression and the efficient amplification. To improve the temporal contrast of the laser pulses, a high-contrast 1-kHz amplifier system is used as a front-end. A grating stretcher which makes the laser pulse have 1-ns duration is used to prevent optical damages due to high pulse energy during amplification. A grating compressor has been designed with group delay analysis to obtain the recompressed pulse duration close to the transform-limited pulse duration. The final laser pulses are expected to have energy above $20 \mathrm{~J}$ and duration below $40 \mathrm{fs}$.
\end{abstract}

Keywords: Ti:sapphire laser, PW laser

OCIS codes : (140.3590) Lasers, titanium; (140.4480) Optical amplifiers; (140.7090) Ultrafast lasers ; (320.7090) Ultrafast lasers

\section{INTRODUCTION}

Ti:sapphire laser systems using chirped-pulse amplification (CPA) can produce optical laser pulses with peak powers of terawatt level and femtosecond pulse durations [1-3]. These high-peak-power laser systems have been used in many high-field applications such as high harmonic generation, optical-field ionization and plasma physics. Recently, the CPA Ti:sapphire laser systems with peak powers of $\mathrm{PW}$ level have been developed by several research groups since the 0.85-PW 33-fs Ti:sapphire laser had been developed at JAERI for the first time [4-8]. These ultra-high-peak-power laser systems are expected to make a significant breakthrough in high-field physics areas such as relativistic nonlinear optics, strong field ionization, and pair plasma production [9]. At APRI a CPA femtosecond 10-Hz 100-TW Ti:sapphire laser has been developed, and then used actively in several ap-

\footnotetext{
*Corresponding author: sungjh@gist.ac.kr

${ }^{\dagger}$ Corresponding author: leejm@gist.ac.kr
}

plication experiments up to now, such as electron generation, proton generation, and x-ray generation $[10,11]$.

In this paper, we present the generation of femtosecond 0.5-PW laser pulses through upgrading the $10-\mathrm{Hz} 100-\mathrm{TW}$ Ti:sapphire laser at APRI and the optimization of the temporal qualities of the laser pulses. A booster amplifier in which a pulse energy can be over $30 \mathrm{~J}$ has been designed and analyzed. A parasitic lasing which usually occurs in the large aperture amplifiers such as our booster amplifier has been investigated and a method for the parasitic lasing suppression has also been investigated. We have estimated the output energy of the booster amplifier by optimizing the beam diameter and the number of passes with a Frantz-Nodvic simulation [12]. A 1-kHz amplifier system with a high contrast ratio is installed as a new front-end to improve the temporal contrast ratio for the application experiments, and a stretcher has been designed to have a longer pulse during amplification for preventing optical damage due to the higher pulse energy, as compared with the pulse energy of the 


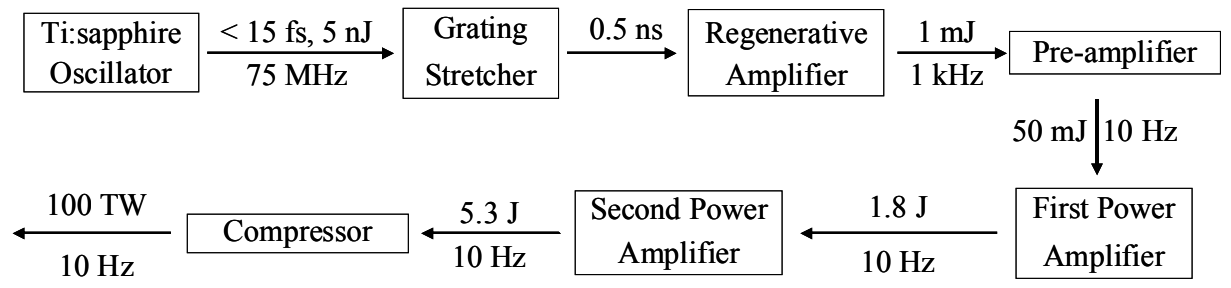

FIG. 1. Schematics of the 10-Hz 100-TW Ti:sapphire laser at APRI.



(a)

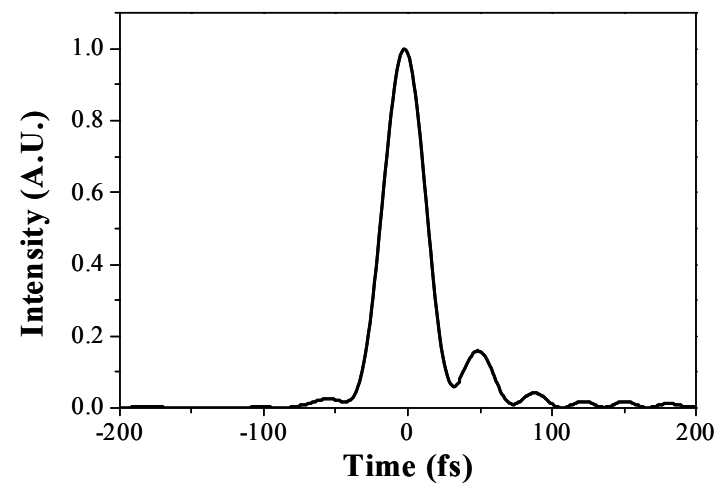

(b)

FIG. 2. The spectral intensity (a) and the temporal intensity (b) of the laser pulse after the compressor in the $10-\mathrm{Hz}$ 100-TW Ti:sapphire laser.

100-TW laser. Finally, we have designed a compressor so that the recompressed pulse duration can be optimized. We have found the optimized condition of the compressor by analyzing the group delay of the laser system.

\section{10-Hz 100-TW TI:SAPPHIRE LASER AT APRI}

The CPA 10-Hz 100-TW Ti:sapphire laser at APRI consists of a laser oscillator, a grating stretcher, a regenerative amplifier, a pre-amplifier, two power amplifiers and a grating compressor, as shown in Fig. 1.

A femtosecond Ti:sapphire laser oscillator generates 15 -fs pulses with an energy of $5 \mathrm{~nJ}$ at $3.0-\mathrm{W}$ pump power. Laser pulses from the oscillator are stretched to about $0.5 \mathrm{~ns}$ to prevent optical damage during amplification by an aberration-free all-reflective Öffner-triplet -type stretcher, in which a grating with 1400 grooves/mm is used. Laser pulses then pass through an acousto-optic programmable dispersive filter (Dazzler, Fastlite), which can control the spectral amplitude and phase of the laser pulses simultaneously to compensate for the gain narrowing and the high-order dispersions, and through a Faraday isolator that blocks the backward amplified spontaneous emission (ASE) from the amplifiers. The laser pulse is then injected into a regenerative amplifier and its energy is boosted up to $1.0 \mathrm{~mJ}$ with a $10-\mathrm{mJ}$ pump energy. The amplified pulse from the regenerative amplifier is injected into a 4-pass pre-amplifier. When pumped by 150-mJ green laser pulses, the amplified pulse energy reaches $50 \mathrm{~mJ}$. The amplified pulse from the pre -amplifier is upcollimated to $12-\mathrm{mm}$ diameter and then injected into a 4-pass power amplifier. When the 4-pass power amplifier is pumped by green laser pulses with 4.0-J energy at $10 \mathrm{~Hz}$, the output pulse energy is $1.8 \mathrm{~J}$. The output pulses from the 4-pass power amplifier are upcollimated to 22-mm diameter and then injected into a 2-pass power amplifier. When the second power amplifier is pumped by green laser pulses with $8.8-\mathrm{J}$ energy at $10 \mathrm{~Hz}$, the output pulse energy is increased into $5.3 \mathrm{~J}$. The amplified pulses from the second power amplifier are upcollimated to 70-mm diameter to prevent the optical damage to gratings in a compressor and then doublepassed through two parallel gratings that have 1480 grooves $/ \mathrm{mm}$. The spectral and the temporal intensities of the recompressed laser pulse are shown in Fig. 2. Here, the spectral width (FWHM) is $48 \mathrm{~nm}$ and the pulse duration is $33 \mathrm{fs}$. After compression, the output energy is $3.4 \mathrm{~J}$, giving a peak power of $100 \mathrm{TW}$. The temporal contrast of the compressed laser pulse is measured with a commercial third-order cross-correlator (Sequoia, Amplitude Technologies). Figure 3 shows that the ASE background level is $2 \times 10^{-7}$ comparing the peak of the main pulse in a-few-tens ps range. This ASE background level is not low enough to prevent the production of a preplasma by the ASE if the laser beam is focused with intensity above $10^{20} \mathrm{~W} / \mathrm{cm}^{2}$ on a target in the application experiments. 


\section{0.1-Hz 0.5-PW TI:SAPPHIRE LASER AT APRI}

A CPA femtosecond Ti:sapphire laser which is upgraded from the 10-Hz 100-TW Ti:sapphire laser has been designed to generate $0.1-\mathrm{Hz}$ 0.5-PW laser pulses with temporal optimization. One method to increase the peak power of the laser pulse is to increase the pulse energy by adding another amplifier. Therefore, a high-energy booster amplifier is added to obtain a pulse energy above $30 \mathrm{~J}$ which is needed to reach $0.5-\mathrm{PW}$ peak power. To improve the temporal contrast of the $0.5-\mathrm{PW}$ laser pulse, a 1-kHz multi-pass amplifier system is used instead of the regenerative amplifier of the 100-TW laser. A stretcher and a compressor, which have been designed to prevent optical damage and optimize the pulse duration in the 0.5 -PW laser, are used instead of the stretcher and compressor in the 100-TW laser. Consequently, the 0.5-PW Ti:sapphire laser consists of a $1-\mathrm{kHz}$ multi-pass amplifier system, a grating stretcher, a preamplifier, two power amplifiers, a booster amplifier and a grating compressor, as shown in Fig. 4. The pre -amplifier and two power amplifiers used in the 100-TW Ti:sapphire laser are also used in the 0.5-PW Ti:sapphire laser as they are.

In large aperture amplifiers such as the booster amplifier of the 0.5-PW laser, the laser gain and the output energy are usually limited by transverse parasitic lasing [13]. The parasitic lasing is generated from the formation of a transverse laser cavity by Fresnel reflection at the edges of the crystal. One method to suppress this parasitic

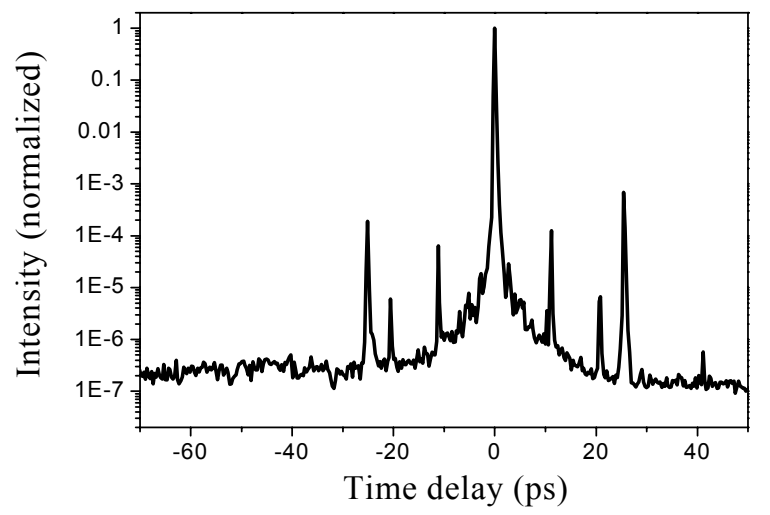

FIG. 3. The measured third-order cross-correlation trace of the laser pulse after the compressor in the $10-\mathrm{Hz} 100$ -TW Ti:sapphire laser. lasing is to reduce the Fresnel reflection at the crystal edges by using cladding materials whose refractive index is matched closely to that of the crystal, 1.76. Indexmatched polymer thermoplastic and index-matched liquid have been successfully demonstrated as cladding materials [4-8]. Our booster amplifier contains a 100-mm-diameter and 25-mm-long Ti:sapphire crystal with an absorption coefficient of $1.2 \mathrm{~cm}^{-1}$. In our laser system, 1-bromonaphthalene with a refractive index of 1.66 is used as an index-matched liquid since it is easily available, not corrosive, and able to be circulated around the crystal, if necessary, for crystal cooling [8]. This liquid will reduce the Fresnel reflection coefficient at the crystal edges from $2.0 \%$ with distilled water to $0.086 \%$. The parasitic lasing will be suppressed if the transverse gain is less than 1200 with the Fresnel reflection of $0.086 \%$. The calculated result shows that the pump beam diameter should be more than $67 \mathrm{~mm}$ to reduce the transverse gain below 1200 with a total pump energy of $80 \mathrm{~J}$. Figure 5 (a) shows the transverse gain as a function of position along the crystal when the crystal is pumped by equal pump energy $(40 \mathrm{~J})$ and beam diameter $(67 \mathrm{~mm})$ on its two faces. The maximum transverse gain is 1200 at the crystal faces. Figure 5 (b) shows the minimum beam diameter to suppress the parasitic lasing at given Fresnel reflection. A laser dye IR 140 is dissolved in the index-matched liquid to absorb the transmitted amplified spontaneous emission that generates transverse lasing by reflections on an inner wall of the crystal mount [8].

A booster amplifier has been designed to obtain the pulse energy above $30 \mathrm{~J}$. The booster amplifier is pumped by Nd:glass laser system (Continuum). The pump laser system produces $527-\mathrm{nm}$ laser pulses at $0.1-\mathrm{Hz}$ repetition rate in twelve beams, each of which has energy of 8.5 J. Although the maximum energy of the Nd:glass pump laser system is about $100 \mathrm{~J}$, only $80-\mathrm{J}$ energy is usually used for the safety of the Nd:glass laser. To estimate the output energy in the booster amplifier, we used Frantz-Nodvic simulation. Figure 6 shows the simulation result of the output energy as a function of beam diameter after each pass with a pump energy of $80 \mathrm{~J}$. The simulation result shows that we can obtain 41-J output energy with $67-\mathrm{mm}$ beam diameter after 4 passes while suppressing the parasitic lasing.

A commercial $1-\mathrm{kHz}$ amplifier system is installed as

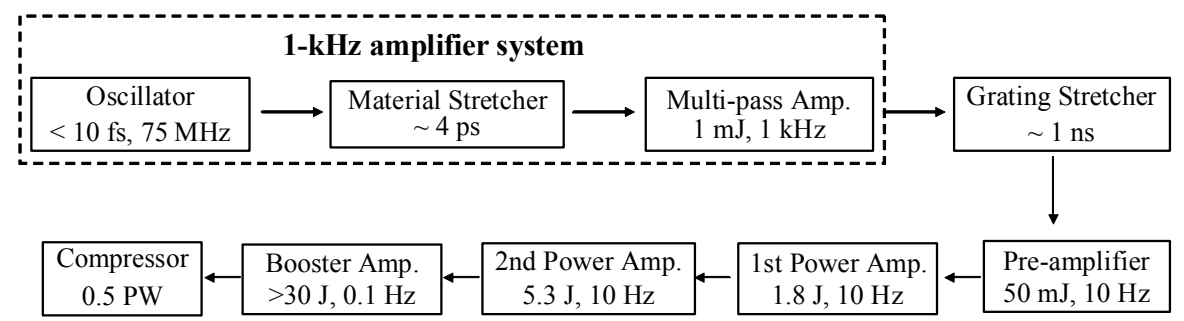

FIG. 4. Schematics of the CPA femtosecond 0.1-Hz 0.5-PW Ti:sapphire laser at APRI. 


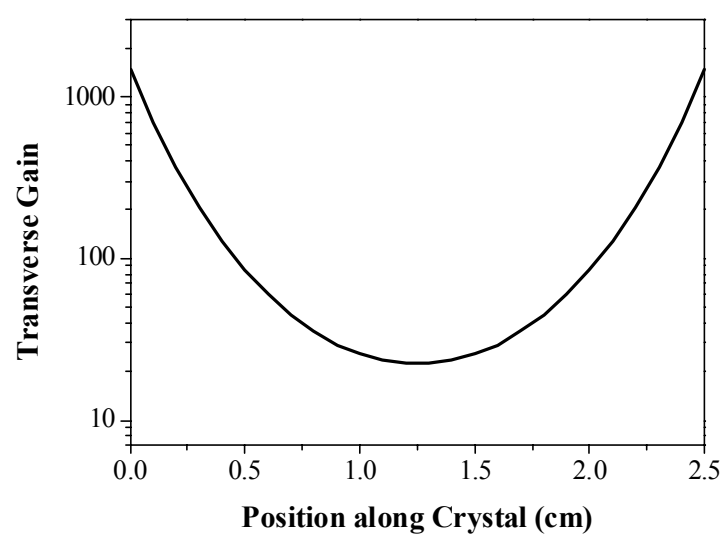

(a)

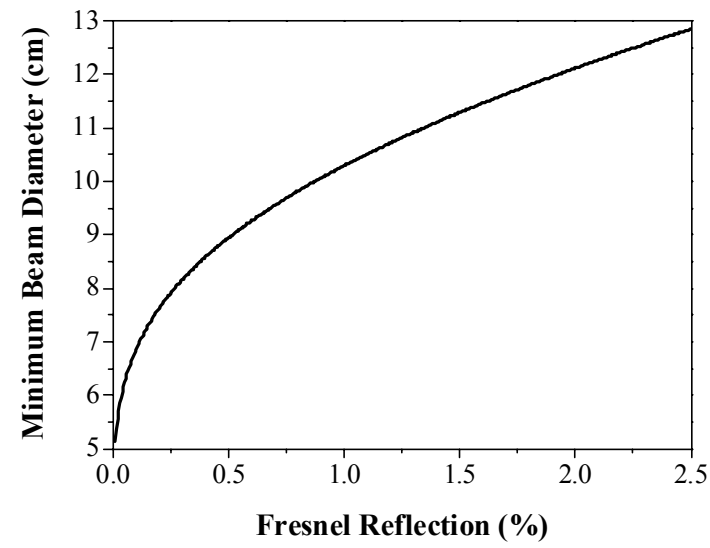

(b)

FIG. 5. Transverse gain along the crystal (a) and minimum beam diameter to suppress parasitic lasing as a function of Fresnel reflection coefficient (b).

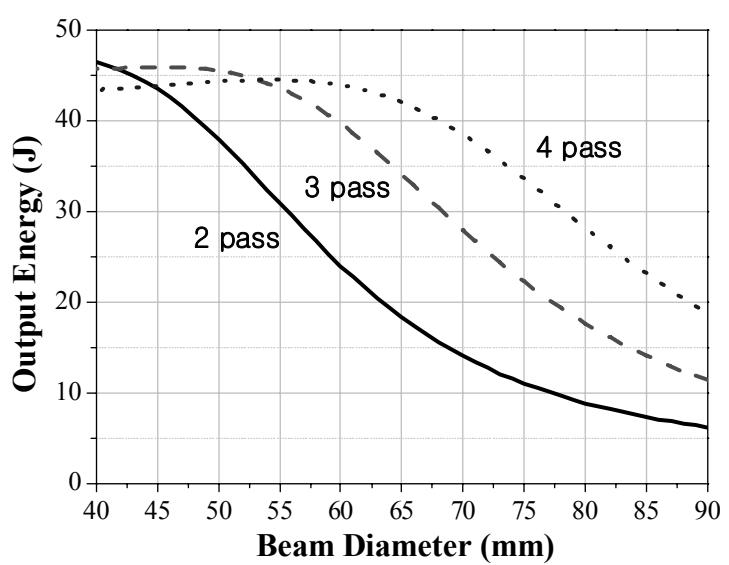

FIG. 6. Calculated output energy as a function of beam diameter after each pass in the booster amplifier.

a new front-end to enhance the temporal contrast of the 0.5-PW laser pulse by providing high-contrast pulses as seed pulses for the amplifiers. For laser-matter interaction applications, the laser beam is focused with intensity above $10^{20} \mathrm{~W} / \mathrm{cm}^{2}$ on a target. Therefore, the laser pulse is required to have high contrast ratio above $10^{9}: 1$, to prevent the production of a preplasma by prepulses such as ASE before the main pulse reaches at the target. The ASE background of the APRI 100-TW laser was generated mostly in the regenerative amplifier. Since a multi-pass amplifier is generally preferred to a regenerative amplifier for enhancement of the temporal contrast, the $1-\mathrm{kHz}$ multi-pass amplifier is installed instead of the regenerative amplifier in the $0.5-\mathrm{PW}$ laser. The commercial 1-kHz amplifier system consists of a Ti:sapphire oscillator, a material stretcher and a $1-\mathrm{kHz}$ Ti:sapphire multi-pass amplifier (Femtopower Compact Pro, Femtolasers). The ASE background level of the $1-\mathrm{kHz}$ amplified pulse after compression is $1 \times 10^{-10}$ in a-few-tens of ps range. The temporal contrast of the 0.5 -PW laser pulse is expected to be enhanced by pro- viding these high-contrast pulses as seed pulses for the amplifiers.

A grating stretcher for the $0.5-\mathrm{PW}$ laser has been designed so that the laser pulse duration can be made to be long enough to suppress optical damages during amplification. To determine the stretched-pulse duration, we have considered nonlinear damage in the material and surface damage of the optical components such as mirrors and lenses. The serious nonlinear damage is caused by self-focusing which is induced by strong nonlinear phase shift. The B integral that is a measure of a cumulative nonlinear phase shift is defined as

$$
\mathrm{B}=\frac{2 \pi}{\lambda_{0}} \int n_{2}(z) I(z) d z
$$

where $\mathrm{z}$ is the position in laser beam direction, $n_{2}$ the nonlinear refractive index of material and $I$ the laser pulse intensity. The B-integral value should be maintained below 1 radian to avoid the nonlinear damage due to 


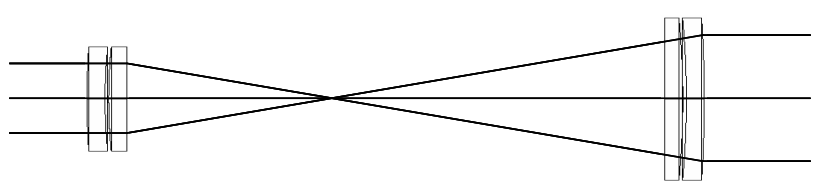

(a)



(b)

FIG. 7. Schematics (a) and chromatic focal shift (b) of the achromatic beam expander to be positioned before the booster amplifier.

self-focusing. We can consider the antireflection (AR) coating damage as a typical surface damage. The damage threshold of a V-type AR coating is actually about 10 $\mathrm{J} / \mathrm{cm}^{2}$ at 8 -ns pulses (CVI laser). We can estimate the surface damage threshold at a given pulse duration by using the empirical scaling law that the damage threshold is proportional to the square root of the pulse duration $[14,15]$. Here, we referred to the above AR-coating damage threshold for estimation of the surface damage threshold at a given pulse duration. If the 0.5-ns-long pulse is used during amplification in the 0.5-PW laser like the 100-TW laser, the B integral and the energy fluence after the booster amplifier will be about $0.5 \mathrm{rad}$ and $1.8 \mathrm{~J} / \mathrm{cm}^{2}$, respectively [16]. The B-integral value of $0.5 \mathrm{rad}$ is tolerable for high power lasers. But, if considering that the surface damage threshold is $2.5 \mathrm{~J} / \mathrm{cm}^{2}$ at 0.5 -ns pulse duration, we can not exclude a possibility that the surface damage happens with the energy fluence of $1.8 \mathrm{~J} / \mathrm{cm}^{2}$. To completely avoid the possibility of surface damage, we should make the pulse duration longer. We have thereupon designed a grating stretcher for the 0.5-PW laser to increase the pulse duration to 1 ns. When the pulse duration is $1 \mathrm{~ns}$, the surface damage threshold is 3.5 $\mathrm{J} / \mathrm{cm}^{2}$. Since this value is two times larger than the energy fluence, we can sufficiently prevent the surface damage. Just like the stretcher of the 100-TW laser, the stretcher of the $0.5-\mathrm{PW}$ laser is an aberration-free all-reflective Öffner-triplet-type stretcher, in which two spherical mirrors and a grating with 1400 grooves/mm are used. To obtain the 1-ns pulse duration, the radii of curvature of the primary spherical mirror and the second spherical mirror are determined to be $1800 \mathrm{~mm}$ and $-900 \mathrm{~mm}$, respectively. And, the optimized incident angle on the grating is $43 \mathrm{deg}$ and the perpendicular separation between the grating and the grating image is $600 \mathrm{~mm}$.

An achromatic beam expander is used instead of a simple beam expander when the laser pulse is upcollimated before the booster amplifier to prevent temporal broadening of the focal spot. The Keplerian-type beam expander is usually used to upcollimate the laser pulse. This type of expander consists of two positive lenses separated by the sum of their focal lengths. The simple beam expander composed of two singlet lenses was used to magnify the laser beam before the compressor in the 100-TW laser. This simple beam expander induces chromatic aberration which reduces the peak intensity of the focal spot and broadens the temporal profile of the focal spot [17]. Therefore, the achromatic doublet lenses composed of BK7 and SF6 material are used instead of singlet lenses to minimize the chromatic aberration. Figure 7 shows that the chromatic focal shift of the expander is very small in the spectral range of $750-850 \mathrm{~nm}$.

A grating compressor has been designed so that the recompressed pulse duration can be optimized. The beam diameter is expanded to $190 \mathrm{~mm}$ before the compressor to eliminate thermal distortion and optical damage on the gratings, taking account of a grating damage threshold of $100 \mathrm{~mJ} / \mathrm{cm}^{2}$. The groove density of the grating is 1480 grooves $/ \mathrm{mm}$ that is different from the groove density of the stretcher, 1400 grooves $/ \mathrm{mm}$. In order to minimize the duration of the recompressed pulse, we have optimized an incident angle on the grating and a distance between two gratings with a genetic algorithm. First, we calculated a group delay induced by the stretcher and materials in the amplifiers. The solid line in Fig. 8 (a) indicates the calculated group delay. The group delay can be compensated for by a group delay induced by the compressor. The genetic algorithm has allowed us to find the incidence angle and the distance which minimize the pulse duration calculated from total group delay after the compressor. The optimized incidence angle and the distance are $50.10 \mathrm{deg}$ and $1002 \mathrm{~mm}$, respectively. The calculated result shows the recompressed pulse duration is close to the transform-limited pulse duration, 29 fs with the spectrum of the 10-Hz 100-TW Ti:sapphire laser shown in Fig. 2 (a). If the compressor throughput efficiency is about $50 \%$, the output energy of the compressor will be about $20 \mathrm{~J}$, giving a peak power above $0.5 \mathrm{PW}$. 

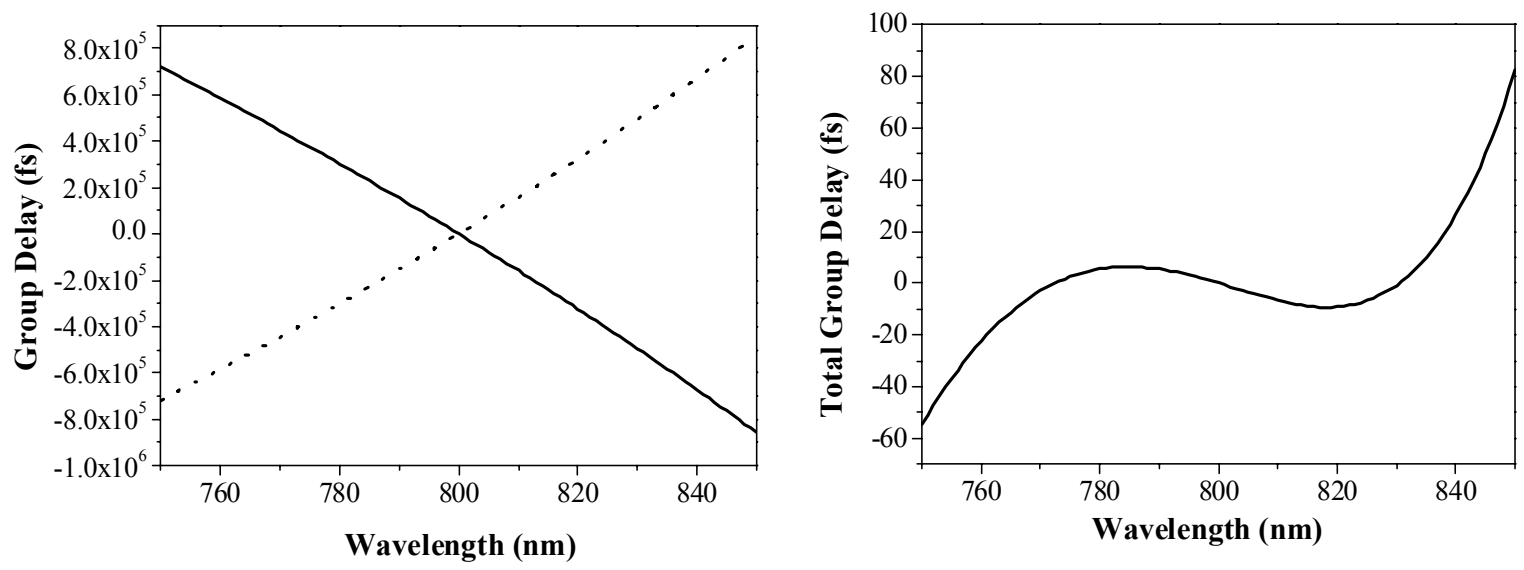

FIG. 8. Group delays induced by the stretcher and materials (solid line) and the compressor (dotted line) (a) and total group delay after the compressor (b).

\section{CONCLUSION}

We have designed a CPA femtosecond Ti:sapphire laser system which generates $0.1-\mathrm{Hz}$ 0.5-PW laser pulses with temporal optimization, using a $10-\mathrm{Hz}$ 100-TW Ti:sapphire laser. A booster amplifier to be added has been designed to obtain pulse energy above $30 \mathrm{~J}$. In the booster amplifier, index-matching liquid is used as a cladding material of the crystal for the parasitic lasing suppression and 41-J output energy is expected with 80-J pump energy and $67-\mathrm{mm}$ beam diameter after 4 passes. The laser pulses with a $10^{10}$ contrast ratio, which are generated by a $1-\mathrm{kHz}$ multi-pass amplifier system, are used as seed pulses of amplifiers in the 0.5-PW laser for temporal contrast enhancement. A stretcher has been designed to obtain the pulse duration of 1 ns for prevention of optical damages. The laser beam is upcollimated through an achromatic doublet beam expander before injected into the booster amplifier to prevent temporal broadening of the focal spot. A compressor has been designed to obtain the recompressed pulse duration close to the transform-limited pulse duration. Final laser pulses are expected to have energy above $20 \mathrm{~J}$ and duration below 40 fs.

\section{ACKNOWLEDGMENT}

This work was supported by the Ministry of Knowledge and Economy of Korea through the Ultrashort Quantum Beam Facility Program.

\section{REFERENCES}

1. J. P. Chambaret, C. Le Blanc, G. Chériaux, P. Curley, G. Darpentigny, P. Rousseau, G. Hamoniaux, A. Antonetti, and F. Salin, "Generation of 25-TW, 32-fs pulses at $10 \mathrm{~Hz}$, Opt. Lett. 21, 1921-1923 (1996).

2. K. Yamakawa, M. Aoyama, S. Matsuoka, T. Kase, Y. Akahane, and H. Takuma, "100-TW sub-20-fs Ti:sapphire laser system operating at a $10-\mathrm{Hz}$ repetition rate," Opt. Lett. 23, 1468-1470 (1998).

3. M. Pittman, S. Ferre, J. P. Rousseau, L. Notebaert, J. P. Chambaret, and G. Cheriaux, "Design and characterization of a near-diffraction-limited femtosecond 100-TW 10-Hz high-intensity laser system," Appl. Phys. B 74, 529-535 (2002).

4. M. Aoyama, K. Yamakawa, Y. Akahane, J. Ma, N. Inoue, H. Ueda, and H. Kiriyama, “0.85-PW, 33-fs Ti:sapphire laser,” Opt. Lett. 28, 1594-1596 (2003).

5. F. Ple, M. Pittman, G. Jamelot, and J.-P. Chambaret, "Design and demonstration of a high-energy booster amplifier for a high-repetition rate petawatt class laser system,” Opt. Lett. 32, 238-240 (2007).

6. X. Liang, Y. Leng, C. Wang, C. Li, L. Lin, B. Zhao, Y. Jiang, X. Lu, M. Hu, C. Zhang, H. Lu, D. Yin, Y. Jiang, X. Lu, H. Wei, J. Zhu, R. Li, and Z. Xu, "Parasitic lasing suppression in high gain femtosecond petawatt Ti:sapphire amplifier," Opt. Exp. 15, 15535-15341 (2007).

7. V. Yanovsky, V. Chvykov, G. Kalinchenko, P. Rousseau, T. Planchon, T. Matsuoka, A. Maksimchuk, J. Nees, G. Cheriaux, G. Mourou, and K. Krushelnick, "Ultra-high intensity-300-TW laser at $0.1 \mathrm{~Hz}$ repetition rate," Opt. Exp. 16, 2109-2114 (2008).

8. K. Ertel, C. Hooker, S. J. Hawkes, B. T. Parry, and J. L. Collier, "ASE suppression in a high energy titanium sapphire amplifier,” Opt. Exp. 16, 8039 -8049 (2008).

9. T. Ditmire, S. Bless, G. Dyer, A. Edens, W. Grigsby, G. Hays, K. Madison, A. Maltsev, J. Colvin, M. J. Edwards, R. W. Lee, P. Patel, D. Price, B. A. Remington, R. Sheppherd, A. Wootton, J. Zweiback, E. Liang, and K. A. Kielty, "Overview of future directions in high energy -density and high-field science using ultra-intense laser," Radiation Physics and Chemistry 70, 535-552 (2004).

10. N. A. M. Hafz, T. M. Jeong, I. W. Choi, S. K. Lee, K. H. Pae, V. V. Kulagin, J. H. Sung, T. J. Yu, K.-H. Hong, T. Hosokai, J. R. Cary, D.-K. Ko, and J. Lee, "Stable generation of $\mathrm{GeV}$-class electron beams from 
self-guided laser- plasma channels," Nat. Photonics 2, 571-577 (2008).

11. H. T. Kim, C.-M Kim, I. W. Choi, H. C. Kang, N. Hafz, S. G. Lee, J. H. Sung, T. J. Yu, K.-H. Hong, T. M. Jeong, Y.-C. Noh, D.-K. Ko, J. Tümmler, P. V. Nickles, W. Sandner, K. A. Janulewicz, and J. Lee, "Characteristics of Ni-like silver x-ray laser pumped by single profiled laser pulse," J. Opt. Soc. Am. B 25, 76-84 (2008).

12. L. M. Frantz and J. S. Nodvik, "Theory of pulse propagation in a laser amplifier,” J. Appl. Phys. 34, 2346-2349 (1963).

13. F. G. Patterson, J. Bonlie, D. Price, and B. White, "Suppression of parasitic lasing in large-aperture Ti:sapphire laser amplifiers," Opt. Lett. 24, 963-965 (1999).

14. D. Du, X. Liu, G. Korn, J. Squier, and G. Mourou,
"Laser-induced breakdown by impact ionization in $\mathrm{SiO}_{2}$ with pulse widths from 7 ns to 150 fs," Appl. Phys. Lett. 64, 3071-3073 (1994).

15. B. C. Stuart, M. D. Feit, A. M. Rubenchik, B. W. Shore, and M. D. Perry, "Laser-induced damage in dielectrics with nanosecond to subpicosecond pulses," Phys. Rev. Lett. 74, 2248-2251 (1995).

16. K.-H. Hong, T. J. Yu, J. H. Sung, I. W. Choi, T. M. Jeong, S. K. Lee, Y.-C. Noh, D.-K. Ko, and J. Lee, "Optical damage evaluation of a CPA Ti:sapphire laser for the safe design of a PW system," in Proc. CLEO/ PR (Korea, 2007), paper WP 061.

17. T. M. Jeong, D.-K. Ko, and J. Lee, "Deformation of the focal spot of an ultrashort high- power laser pulse due to chromatic aberration by a beam expander," J. Korean Phys. Soc. 50, 1767-1773 (2008). 